\title{
ON EULER CHARACTERISTICS ASSOCIATED TO EXCEPTIONAL DIVISORS
}

\author{
WILLEM VEYS
}

\begin{abstract}
Let $k$ be an algebraically closed field and $f \in k\left[x_{1}, \ldots, x_{n+1}\right]$. Fix an embedded resolution $h: X \rightarrow \mathbb{A}^{n+1}$ of $f^{-1}\{0\}$ and denote by $E_{i}, i \in$ $S$, the irreducible components of $h^{-1}\left(f^{-1}\{0\}\right)$ with multiplicity $N_{i}$ in the divisor of $f \circ h$. Put also $\stackrel{\circ}{E}_{i}:=E_{i} \backslash \bigcup_{j \neq i} E_{j}$, and denote by $\chi\left(\stackrel{\circ}{E}_{i}\right)$ its Euler characteristic.

Several conjectures concerning Igusa's local zeta function and the topological zeta function of $f$ motivate the study of Euler characteristics associated to subsets $\bigcup_{i \in T} E_{i}$ of $\bigcup_{i \in S} E_{i}$, which are maximal connected with respect to the property that $d \mid N_{i}$ for all $i \in T$. Here $d \in \mathbb{N}, d>1$. We prove that if $h$ maps $\bigcup_{i \in T} E_{i}$ to a point, then

$$
(-1)^{n} \sum_{i \in T} \chi\left(\stackrel{\circ}{E}_{i}\right) \geqslant 0
$$

This generalizes a well-known result for curves. We also prove some vanishing results concerning the $\chi\left(\stackrel{\circ}{E}_{i}\right)$ for such a maximal connected subset $\bigcup_{i \in T} E_{i}$ and give an application on the above-mentioned zeta functions, yielding some confirmation of the holomorphy conjecture for those zeta functions.
\end{abstract}

\section{INTRODUCTION}

(0.1) Let $f \in \mathbb{C}\left[x_{1}, \ldots, x_{n+1}\right]$ and fix an embedded resolution $h: X \rightarrow \mathbb{A}^{n+1}$ of $f^{-1}\{0\}$. Denote by $E_{i}, i \in S$, the irreducible components of $h^{-1}\left(f^{-1}\{0\}\right)$ with multiplicities $N_{i}$ in the divisor of $f \circ h$. Put also $\stackrel{\circ}{E}_{i}:=E_{i} \backslash \bigcup_{j \neq i} E_{j}$.

$(0.2)$ For curves $(n=1)$ let $k_{i}$ denote the number of intersection points of $E_{i}$ with other $E_{j}, j \in S$. The following is essentially known. (We suppose that no $E_{i}$ is created by a silly blowing up in a smooth point of $f^{-1}\{0\}$.)

Proposition [V, Lemma 2.3]. To any exceptional curve $E_{0}$ with $k_{0}=1$ we can associate $r \geqslant 1$ and a path

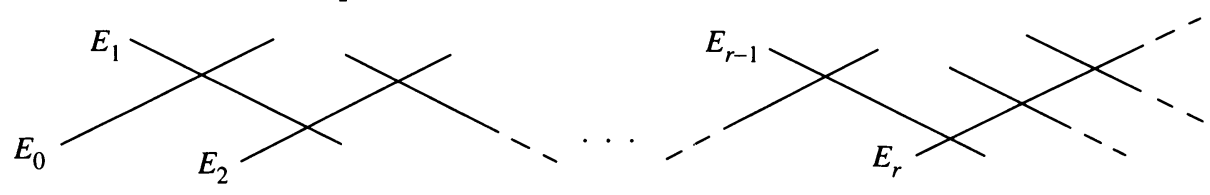

Received by the editors February 271994.

1991 Mathematics Subject Classification. Primary 14E15, 11S40, 32S40.

The author is a senior research assistant of the Belgian National Fund for Scientific Research (N.F.W.O.). 
in the resolution graph, where all $E_{i}$ are exceptional curves, such that

(1) $k_{i}=2$ for $i=1, \ldots, r-1$ and $k_{r} \geqslant 3$; and

(2) $N_{0} \mid N_{i}$ for all $i=1, \ldots, r$.

Suppose for simplicity that $f^{-1}\{0\}$ is reduced. Then the following implies the proposition above.

Assertion. Take some $d \in \mathbb{N}, d>1$. If $\bigcup_{i \in T} E_{i}$ is a maximal connected subset of $\bigcup_{i \in S} E_{i}$ with respect to the property that $E_{i}$ is an exceptional curve and $d \mid N_{i}$ for all $i \in T$, then

$$
\sum_{i \in T} \chi\left(\stackrel{\circ}{E}_{i}\right) \leqslant 0
$$

Here $\chi(\cdot)$ denotes the (complex) Euler-Poincare characteristic. Remark that an exceptional curve $E_{i}$ is isomorphic to $\mathbb{P}^{1}$, so $\chi\left(\stackrel{\circ}{E}_{i}\right)=2-k_{i}$.

(0.3) We will in fact prove the following generalization of $(0.2)$ in all dimensions. Furthermore our proof is conceptual in the sense that it doesn't require $h$ to be a composition of blowing ups.

Theorem (\{3). Suppose that $f^{-1}\{0\}$ is reduced and take $d \in \mathbb{N}, d>1$. If $\bigcup_{i \in T} E_{i} \subset h^{-1}\{0\}$ is a maximal connected subset of $\bigcup_{i \in S} E_{i}$ with respect to the property that $d \mid N_{i}$ for all $i \in T$, then

$$
(-1)^{n} \sum_{i \in T} \chi\left(\stackrel{\circ}{E}_{i}\right) \geqslant 0 .
$$

(The fact that $h$ maps $\bigcup_{i \in T} E_{i}$ to a point implies that all $E_{i}, i \in T$, are proper exceptional varieties.)

A motivation for this theorem are several conjectures relating Igusa's local zeta function and the topological zeta function of $f$ to the monodromy of $f^{-1}\{0\}$. Philosophically $E_{i}$ with $(-1)^{n} \chi\left(\stackrel{\circ}{E}_{i}\right)<0$ are expected to be "compensated' by $E_{i}$ with $(-1)^{n} \chi\left(\stackrel{\circ}{E}_{i}\right)>0$. See [V] for a result for curves.

(0.4) We also prove (in $\S 4)$ some vanishing results concerning the $\chi\left(\stackrel{\circ}{E}_{i}\right)$ for a connected subset $\bigcup_{i \in T} E_{i}$ with $d \mid N_{i}$ for all $i \in T$. Moreover we do not restrict to $\mathbb{C}$ but work simultaneously over a groundfield of arbitrary characteristic.

(0.5) Finally we give an application of those vanishing results on the above mentioned zeta functions. More precisely suppose that $d \in \mathbb{N} \backslash\{0\}$ does not divide the order of any eigenvalue of the local monodromy of $f$ at 0 . Let $\bigcup_{i \in T} E_{i} \subset h^{-1}\{0\}$ be a maximal connected subset of $\bigcup_{i \in S} E_{i}$ with respect to the property that $d \mid N_{i}$ for all $i \in T$. If all $N_{i}, i \in T$, are mutually different and the $E_{i}, i \in T$, satisfy a certain affineness condition, then $\bigcup_{i \in T} E_{i}$ does not contribute to the topological zeta function of $f$ or to 'almost all' Igusa's local zeta functions of $f$. See $\S 5$ for exact statements.

\section{Preliminaries}

(1.1) Let $k$ denote either $\mathbb{C}$ or an algebraic closure $\mathbb{F}_{q}^{a}$ of the finite field with $q$ elements. We fix a nonconstant polynomial $f \in k\left[x_{1}, \ldots, x_{n+1}\right]$, determining a hypersurface $f^{-1}\{0\}$ in $\mathbb{A}^{n+1}(k)$, which we suppose to be reduced. 
See Remark 3.6 for general $f$. Just to simplify notation we also suppose that $f(0)=0$.

Let $h: X \rightarrow \mathbb{A}^{n+1}$ be an embedded resolution (of singularities) of $f^{-1}\{0\}$. (If $k=\mathbb{C}$, then $(X, h)$ always exists for any $f$ by Hironaka's Main Theorem [H]; and if $k=\mathbb{F}_{q}^{a}$ we just suppose some $(X, h)$ to be given. See also $\S 5$ for 'reduction $\bmod P$ ' of a characteristic zero situation.) We denote the (reduced) irreducible components of $h^{-1}\left(f^{-1}\{0\}\right)$ by $E_{i}, i \in S$, and their multiplicities in the divisor of $f \circ h$ by $N_{i}$; i.e. $(f \circ h)=\sum_{i \in S} N_{i} E_{i}$. Now if $k=\mathbb{F}_{q}^{a}$ we furthermore suppose $(X, h)$ to be a tame resolution, i.e. $q$ is prime to $N_{i}$ for each $i \in S$. The $E_{i}$ consist of exceptional varieties and of irreducible components of the strict transform of $f^{-1}\{0\}$; the last ones have $N_{i}=1$.

We put $\stackrel{\circ}{E}_{i}:=E_{i} \backslash \bigcup_{j \neq i} E_{j}, E_{I}:=\bigcap_{i \in I} E_{i}$ and $\stackrel{\circ}{E}_{I}:=E_{I} \backslash \bigcup_{j \notin I} E_{j}$ for any $\varnothing \neq I \subset S$.

Nearby cycles.

(1.2) Fix any prime $\ell \Varangle q$, and let $\mathbb{Q}_{\ell}^{a}$ denote an algebraic closure of the $\ell$-adic numbers. For any scheme $V$ of finite type over $k$ we denote by $H^{*}(V)$ its singular cohomology groups $H^{\bullet}(V, \mathbb{C})$, respectively its $\ell$-adic cohomology groups $H^{\cdot}\left(V, \mathbb{Q}_{\ell}^{a}\right)$.

We abbreviate by $R \Psi$ the complex of nearby cycles $R \Psi_{f \circ h}(\mathbb{C})$ or $R \Psi_{f \circ h}\left(\mathbb{Q}_{\ell}^{a}\right)$ on $h^{-1}\left(f^{-1}\{0\}\right)$ and denote by $\Psi^{j}$ its cohomology sheaves, see [SGA7, Exposés XIII-XIV]. Let also $F_{0}$ denote the (local) Milnorfiber of $f$ at $0\left(\in \mathbb{A}^{n+1}\right)$, and $\mathscr{I}$ the local monodromy group of $\mathbb{A}^{1}(k)$ at 0 . (If $k=\mathbb{C}$, then $\mathscr{I} \cong \mathbb{Z}$.)

The monodromy group $\mathscr{I}$ acts on $F_{0}, R \Psi$ and various associated cohomology objects. By an eigenvalue of monodromy of $f$ at 0 we mean any eigenvalue of the action of $\mathscr{I}$ on any cohomology group $H^{\cdot}\left(F_{0}\right)$. It is well known that those eigenvalues are roots of unity [SGA7, Exposé I].

We also recall the following fundamental facts.

1.3. Proposition [SGA 7, Exposé I, Théorème 3.3], [S, Proposition 6]. Fix any $\varnothing \neq I \subset S$.

(i) All $\Psi^{j}, j \geqslant 0$, are lisse on $\stackrel{\circ}{E}_{I}$.

(ii) There exists an isomorphism

$$
\Psi^{j} \cong \Psi^{0} \otimes \bigwedge^{j} M_{I}
$$

on $\stackrel{\circ}{E}_{I}$, compatible with the action of $\mathscr{I}$. Here $M_{I}$ is the kernel of the linear map $\left(z_{i}\right)_{i \in I} \mapsto \sum_{i \in I} N_{i} z_{i}$ on $\mathbb{C}^{I}$ or $\left(\mathbb{Q}_{\ell}^{a}\right)^{I} ;$ so $\operatorname{dim} M_{I}=|I|-1$.

(iii) For any (closed) point $s \in \stackrel{\circ}{E}_{I}$ we have that

$$
\Psi_{s}^{0} \cong \mathbb{C}^{D_{I}} \text {, respectively } \Psi_{s}^{0} \cong\left(\mathbb{Q}_{\ell}^{a}\right)^{D_{I}},
$$

where $D_{I}$ is a finite set on which $\mathscr{I}$ acts transitively, and $\left|D_{I}\right|$ is equal to the largest common divisor of the $N_{i}, i \in I$.

Remark. The isomorphism in (ii) is not canonical; it becomes canonical if instead of $M_{I}$ we write its Tate twist $M_{I}(-1)$. We will not use this more general statement. 

that

(1.4) Since $h$ is proper and birational we have by [SGA7, Exposé XIII 2.1.7.1]

$$
H^{i}\left(F_{0}\right) \cong \mathbb{H}^{i}\left(h^{-1}\{0\}, R \Psi\right) \text {. }
$$

Isotopic components.

(1.5) For any $d \in \mathbb{N}$ with $d>1$, and in positive characteristic also with $d$ prime to $q$, we choose a character $\varkappa: \mathscr{I} \rightarrow \mathbb{C}^{\times}$, respectively $\varkappa: \mathscr{I} \rightarrow\left(\mathbb{Q}_{\ell}^{a}\right)^{\times}$ of order $d$.

(1.6) For any finite-dimensional $\mathbb{C}$-, respectively $\mathbb{Q}_{\ell}^{a}$-vectorspace, constructible sheaf, or (eventually shifted) perverse sheaf $\mathscr{F}$ on which $\mathscr{I}$ acts we denote by $\mathscr{F} \varkappa$ the $\varkappa$-unipotent part of $\mathscr{F}$, i.e. the maximal subobject in the corresponding category on which $\mathscr{I}$ acts like $\varkappa$ times a unipotent action. One can show that for (perverse) sheaves taking the $\varkappa$-unipotent part commutes with taking (hyper-)cohomology groups.

(1.7) We motivate this construction by the following strategy. If $d \mid N_{i}$ for all $i \in T \subset S$ we will prove in Proposition 2.2 that

$$
\sum_{i \in T} \chi\left(\stackrel{\circ}{E}_{i}\right)=\sum_{i}(-1)^{i} \operatorname{dim} \mathbb{H}^{i}\left(\bigcup_{i \in T} E_{i}, R \Psi^{\varkappa}\right) .
$$

Then the crucial result (Corollary 3.4) is that, when $T$ satisfies the conditions of our main theorem, we have

$$
\mathbb{H}^{i}\left(\bigcup_{i \in T} E_{i}, R \Psi^{\varkappa}\right)=0 \quad \text { if } \quad i \neq n .
$$

\section{EULER CHARACTERISTICS AND HYPERCOHOMOLOGY}

From now on we fix $d \in \mathbb{N}$ as in (1.5) and its associated character $\varkappa$.

2.1. Lemma. (i) We have that $\left(\Psi^{j}\right)^{\varkappa}=0$ on $\bigcup_{d \backslash N_{i}} E_{i}$ for all $j \geqslant 0$.

(ii) On any $E_{I}$ for which $d \mid N_{i}$ for all $i \in I$, we have that $\left(\Psi^{0}\right)^{\varkappa}$ is lisse of rank one and that

$$
\left(\Psi^{j}\right)^{\varkappa} \cong\left\{\begin{array}{cl}
{\left[\left(\Psi^{0}\right)^{\varkappa}\right]^{(|I|-1)}{ }_{j}} & \text { if } j \leqslant|I|-1 \\
0 & \text { if } j>|I|-1 .
\end{array}\right.
$$

Proof. Using the notation of Proposition 1.3(iii) we clearly have that $d X\left|D_{I}\right| \Leftrightarrow$ $E_{I} \subset \bigcup_{d N_{i}} E_{i}$. Since $\mathscr{I}$ acts transitively on $D_{I}$, we have that $\left(\Psi^{0}\right)^{\varkappa}=0$ on any $E_{I} \subset \bigcup_{d \chi N_{i}} E_{i}$ and that the rank of $\left(\Psi^{0}\right)^{\varkappa}$ is one on any $E_{I}$ as in (ii).

The statements for $j>0$ follow immediately from Proposition 1.3(ii).

Further on $\chi(.,$.$) and \chi_{\mathrm{c}}(.,$.$) denote respectively ordinary and compactly$ supported Euler-Poincaré characteristic; in particular $\chi()=.\chi(., \mathbb{C})$ or $\chi()=$. $\chi\left(., \mathbb{Q}_{\ell}^{a}\right)$.

2.2. Proposition. If $d \mid N_{i}$ for all $i \in T$, then

$$
\sum_{i \in T} \chi\left(\stackrel{\circ}{E}_{i}\right)=\sum_{i}(-1)^{i} \operatorname{dim} \mathbb{H}^{i}\left(\bigcup_{i \in T} E_{i}, R \Psi^{\varkappa}\right) .
$$


Proof. Denote $M:=\bigcup_{i \in T} E_{i}$. The ( $\varkappa$-unipotent part of the) spectral sequence of hypercohomology yields

$$
\sum_{i}(-1)^{i} \operatorname{dim} \mathbb{H}^{i}\left(M, R \Psi^{\varkappa}\right)=\sum_{i, j}(-1)^{i+j} \operatorname{dim} H^{i}\left(M,\left(\Psi^{j}\right)^{\varkappa}\right) .
$$

We partition $M$ into the locally closed subsets $\stackrel{\circ}{E}_{\langle k\rangle}:=\bigcup_{I \subset T,|I|=k} \stackrel{\circ}{E}_{I}$. Then by Lemma 2.1 we can describe $\left(\Psi^{j}\right)^{\varkappa}$ on $M$ as

$$
\left.\left(\Psi^{j}\right)^{\varkappa}\right|_{E_{\langle k+1\rangle}} \cong \begin{cases}{\left[\left(\Psi^{0}\right)^{\varkappa}\right]^{\left(\begin{array}{l}
k \\
j
\end{array}\right)}} & \text { if } 0 \leqslant j \leqslant k, \\
0 & \text { if } 0 \leqslant k<j .\end{cases}
$$

So $\chi\left(M,\left(\Psi^{j}\right)^{\varkappa}\right)=\sum_{k \geqslant j} \chi_{c}\left(\stackrel{\circ}{E}_{\langle k+1\rangle},\left(\Psi^{j}\right)^{\varkappa}\right)=\sum_{k \geqslant j}\left(\begin{array}{l}k \\ j\end{array}\right) \chi_{c}\left(\stackrel{\circ}{E}_{\langle k+1\rangle},\left(\Psi^{0}\right)^{\varkappa}\right)$. Then

$$
\begin{aligned}
\sum_{i, j}(-1)^{i+j} \operatorname{dim} H^{i}\left(M,\left(\Psi^{j}\right)^{\varkappa}\right) & =\sum_{j}(-1)^{j} \sum_{k \geqslant j}\left(\begin{array}{c}
k \\
j
\end{array}\right) \chi_{c}\left(\stackrel{\circ}{E}_{\langle k+1\rangle},\left(\Psi^{0}\right)^{\varkappa}\right) \\
& =\sum_{k \geqslant 0}\left[\sum_{j=0}^{k}(-1)^{j}\left(\begin{array}{c}
k \\
j
\end{array}\right)\right] \chi_{c}\left(\stackrel{\circ}{E}_{\langle k+1\rangle},\left(\Psi^{0}\right)^{\varkappa}\right) \\
& =\chi_{c}\left(\stackrel{\circ}{E}_{\langle 1\rangle},\left(\Psi^{0}\right)^{\varkappa}\right) .
\end{aligned}
$$

Since $\left(\Psi^{0}\right)^{\varkappa}$ is lisse of rank one on $\stackrel{\circ}{E}_{\langle 1\rangle}=\bigcup_{i \in T} \stackrel{\circ}{E}_{i}$ we have that

$$
\chi_{c}\left(\stackrel{\circ}{E}_{\langle 1\rangle},\left(\Psi^{0}\right)^{\varkappa}\right)=\chi_{c}\left(\bigcup_{i \in T} \stackrel{\circ}{E}_{i}\right) \text {. }
$$

Using Poincaré duality we finally obtain

$$
\sum_{i, j}(-1)^{i+j} \operatorname{dim} H^{i}\left(M,\left(\Psi^{j}\right)^{\varkappa}\right)=\sum_{i \in T} \chi\left(\stackrel{\circ}{E}_{i}\right) .
$$

Combining (i) and (ii) we are done.

\section{VANISHING OF HYPERCOHOMOLOGY}

(3.1) We now fix the data for Theorem 3.5.

Let $M:=\bigcup_{i \in T} E_{i} \subset h^{-1}\{0\}$ be a maximal connected subset of $\bigcup_{i \in S} E_{i}$ with respect to the property that $d \mid N_{i}$ for all $i \in T$. Remark that then all $E_{i}, i \in T$, must be proper exceptional varieties.

3.2. Lemma. $\mathbb{H}^{i}\left(M, R \Psi^{\varkappa}\right)=0$ for $i>n$.

Proof. Since $M$ is maximal, we have that $d \not N_{j}$ for all $E_{j}, j \in S \backslash T$, which have a nonempty intersection with $M$. Then by Lemma 2.1(i) it is clear that $\left(\Psi^{j}\right)^{\varkappa}=0$ on $M \backslash \bigcup_{\varnothing \neq I \subset T} \stackrel{\circ}{E}_{I}=M \cap \bigcup_{j \in S \backslash T} E_{j}$ for all $j \geqslant 0$. So the spectral sequence of hypercohomology yields

$$
\mathbb{H}^{i}\left(M \backslash \bigcup_{\varnothing \neq I \subset T} \stackrel{\circ}{E}_{I}, R \Psi^{\varkappa}\right)=0 \quad \text { for all } i \geqslant 0 .
$$


Applying the Mayer-Vietoris sequence for $h^{-1}\{0\}=M \cup\left(h^{-1}\{0\} \backslash \bigcup_{\varnothing \neq I \subset T} \stackrel{\circ}{E}_{I}\right)$ we obtain

$$
\mathbb{H}^{i}\left(M, R \Psi^{\varkappa}\right) \subset \mathbb{H}^{i}\left(h^{-1}\{0\}, R \Psi^{\varkappa}\right)=H^{i}\left(F_{0}\right)^{\varkappa}
$$

(1.4), which proves the lemma since $H^{i}\left(F_{0}\right)=0$ for $i>n$; see for example [SGA7, Exposé I, Théorème 4.2].

Remark. This result generalizes an idea of Denef; the special case where $|T|=1$ is mentioned in the proof of [D4, Lemma 5.1].

3.3. Proposition. $\check{H}^{i}\left(M, R \Psi^{\varkappa}\right) \cong \mathbb{H}^{2 n-i}\left(M, R \Psi^{\varkappa^{-1}}\right)$. (Here denotes taking the dual vector space.)

Proof. Let $\Pi: M \rightarrow$ Spec $k$ denote the structure map of $M$ and $\varepsilon$ the injection $M \hookrightarrow h^{-1}\left(f^{-1}\{0\}\right)$. By definition of hypercohomology we have that

$$
\check{H}^{i}\left(M, R \Psi^{\varkappa}\right)=\check{H}^{i}\left(R \Pi_{*} \varepsilon^{*} R \Psi^{\varkappa}\right) \text {. }
$$

This is tautologically isomorphic to

$$
H^{-i}\left(D R \Pi_{*} \varepsilon^{*} R \Psi^{\varkappa}\right) \cong H^{-i}\left(R \Pi_{*} R \varepsilon^{!} D R \Psi^{\varkappa}\right),
$$

where $D$ denotes the Verdier dual ${ }^{1}$ in the appropriate derived categories. Now $D R \Psi \simeq R \Psi[2 n]$ (see for example [B] over $\mathbb{C}$ and [Il, Théorème 4.2] for the proof of $\mathrm{O}$. Gabber in positive characteristic) and then it is not difficult to verify that

$$
D R \Psi^{\varkappa} \simeq R \Psi^{\varkappa^{-1}}[2 n] .
$$

Furthermore we claim that

$$
R \varepsilon^{!} R \Psi^{\varkappa^{-1}} \simeq \varepsilon^{*} R \Psi^{\varkappa^{-1}}
$$

Then $\check{H}^{i}\left(M, R \Psi^{\varkappa}\right) \cong H^{-i}\left(R \Pi_{*} \varepsilon^{*} R \Psi^{\varkappa-1}[2 n]\right) \cong H^{2 n-i}\left(R \Pi_{*} \varepsilon^{*} R \Psi^{\varkappa^{-1}}\right)$, which is by definition $\mathbb{H}^{2 n-i}\left(M, R \Psi^{\varkappa^{-1}}\right)$.

Proof of claim $(*)$. We decompose $\varepsilon$ as

$$
M \stackrel{\alpha}{\hookrightarrow} M \cup\left(\bigcup_{s \in J} \stackrel{\circ}{E}_{s}\right) \stackrel{\beta}{\hookrightarrow} h^{-1}\left(f^{-1}\{0\}\right),
$$

where $J=\left\{s \in S \mid s \notin T\right.$ and $\left.E_{s} \cap M \neq \varnothing\right\}$ ranges over the $E_{s}$ that intersect $M$. Then $\alpha$ is closed and $\beta$ is open. So

$$
R \varepsilon^{!} R \Psi^{\varkappa^{-1}} \simeq R \alpha^{!} \beta^{*} R \Psi^{\varkappa^{-1}} .
$$

We now consider (in the derived category of complexes of sheaves with bounded constructible cohomology) the exact triangle

$$
\alpha_{*} R \alpha^{!} K \longrightarrow K \longrightarrow R j_{*} j^{*} K \stackrel{+1}{\longrightarrow}
$$

associated to $K:=\beta^{*} R \Psi^{\varkappa^{-1}}$ and $M \stackrel{\alpha}{\leftrightarrow} M \cup\left(\bigcup_{s \in J} \stackrel{\circ}{E_{s}}\right) \stackrel{j}{\hookleftarrow} \bigcup_{s \in J} \stackrel{\circ}{E_{s}}$. By Lemma 2.1(i) we have that $j^{*} K \simeq 0$. So $\alpha_{*} R \alpha^{!} K \simeq K$, which implies that $R \alpha^{!} K \simeq$ $\alpha^{*} K$, i.e.

$$
R \alpha^{!} \beta^{*} R \Psi^{\varkappa^{-1}} \simeq \varepsilon^{*} R \Psi^{\varkappa^{-1}}
$$

${ }^{1}$ Concretely on any scheme $V$ of finite type over $k$ with structure map $\pi: V \rightarrow$ Spec $k$ the Verdier dual is defined as $D(-):=\mathrm{R} \operatorname{Hom}\left(-, R \pi^{!} k\right)$. With this normalization we have on a smooth $V$ that $D k=k[2 \operatorname{dim} V]$. 
The claim follows from (i) and (ii).

3.4. Corollary. $\mathbb{H}^{i}\left(M, R \Psi^{\varkappa}\right)=0$ for $i \neq n$.

Proof. This is immediate from Proposition 3.3 and Lemma 3.2, since the last is obviously also valid for $\varkappa^{-1}$.

Joining this corollary to Proposition 2.1 we obtain our positivity theorem.

3.5. Theorem. Take $d \in \mathbb{N}$ with $d>1$, and in positive characteristic also with $d$ prime to $q$. If $\bigcup_{i \in T} E_{i} \subset h^{-1}\{0\}$ is a maximal connected subset of $\bigcup_{i \in S} E_{i}$ with respect to the property that $d \mid N_{i}$ for all $i \in T$, then

$$
(-1)^{n} \sum_{i \in T} \chi\left(\stackrel{\circ}{E}_{i}\right) \geqslant 0 .
$$

3.6. Remark. (i) If $f=0$ is not necessarily reduced let $f=\prod_{j \in J} f_{j}^{N_{j}}$ be the decomposition of $f$ in irreducible elements. Then all our preceding and forthcoming results remain true if instead of $d>1$ we take $d$ such that it does not divide any $N_{j}, j \in J$, for which $0 \in\left\{f_{j}=0\right\}$.

(ii) We can extend our results further to the following data : replace $\mathbb{A}^{n+1}$ by any nonsingular variety $V$ and $f$ by a nonconstant regular function on $V$.

(3.7) Theorem 3.5 is in general not true if $\operatorname{dim} h\left(\bigcup_{i \in T} E_{i}\right)>0$; now in view of connections with monodromy it is more natural to study the $\chi\left(\stackrel{\circ}{E}_{i} \cap h^{-1}\{0\}\right)$; see [A, Theorem 3].

Consider for example the easy case where $f$ depends only on $x_{1}, \ldots$, $x_{n+1-r}$; say $f\left(x_{1}, \ldots, x_{n+1}\right)=g\left(x_{1}, \ldots, x_{n+1-r}\right)$. Via the natural embedding $\mathbb{A}^{n+1-r} \hookrightarrow \mathbb{A}^{n+1}$ we have

$$
g^{-1}\{0\}=f^{-1}\{0\} \cap \mathbb{A}^{n+1-r} \quad \text { and } \quad f^{-1}\{0\} \cong g^{-1}\{0\} \times \mathbb{A}^{r} .
$$

We now suppose that the resolution $(X, h)$ of $f^{-1}\{0\}$ is derived in the obvious way from a resolution $\left(X^{\prime}, h^{\prime}\right)$ of $g^{-1}\{0\}$ with associated irreducible components $E_{i}^{\prime}, i \in T$, of $h^{\prime-1}\left(g^{-1}\{0\}\right)$. So in particular

$$
\begin{array}{ll}
X^{\prime}=X \cap h^{-1}\left(\mathbb{A}^{n+1-r}\right), & X \cong X^{\prime} \times \mathbb{A}^{r}, \\
E_{i}^{\prime}=E_{i} \cap X^{\prime}, & E_{i} \cong E_{i}^{\prime} \times \mathbb{A}^{r} .
\end{array}
$$

Proposition. Take $d$ as in Theorem 3.5 and let $\bigcup_{i \in T} E_{i}$ be a maximal connected subset of $\bigcup_{i \in S} E_{i}$ with respect to the property that $d \mid N_{i}$ for all $i \in T$. If $\bigcup_{i \in T} E_{i}^{\prime} \subset h^{\prime-1}\{0\}$, then

$$
(-1)^{n-r} \sum_{i \in T} \chi\left(\stackrel{\circ}{E}_{i} \cap h^{-1}\{0\}\right) \geqslant 0 .
$$

Proof. Since $h^{-1}\{0\} \subset X^{\prime}$, we have for all $i \in T$ that $\stackrel{\circ}{E}_{i} \cap h^{-1}\{0\}=\stackrel{\circ}{E}_{i}^{\prime} \cap$ $h^{\prime-1}\{0\}=\stackrel{\circ}{E}_{i}^{\prime}$. Then the statement is just Theorem 3.5 applied to $g,\left(X^{\prime}, h^{\prime}\right)$ and $\bigcup_{i \in T} E_{i}^{\prime}$.

One can eventually generalize these ideas to the case where 0 has an open neighbourhood $V$ in $\mathbb{A}^{n+1}$ which satisfies (compatible) isomorphisms

$$
V \cong B \times I \quad \text { and } \quad f^{-1}\{0\} \cap V \cong\left(f^{-1}\{0\} \cap B\right) \times I
$$

for some 'transversal slice' $B$ of $V$. 


\section{Nullity OF EULER CHARACTERISTICS}

We still fix $d \in \mathbb{N}$ as in (1.5) and its associated character $\varkappa$.

(4.1) We say that an exceptional variety $E_{j}, j \in S$, for which $d \mid N_{j}$ satisfies the affineness condition if $E_{j} \backslash \bigcup_{d \chi_{i}} E_{i}$ is affine.

Here we should remark that in concrete examples and for fixed $d$ a lot of exceptional varieties satisfy this affineness condition. It is neither a generic nor a special condition but something 'in between'.

(4.2) We have in the definition above that the restriction of $\left(\Psi^{0}\right)^{\varkappa}$ to $E_{j}$ is a local system on $E_{j} \backslash \bigcup_{d \chi_{i}} E_{i}$ and vanishes elsewhere. When $E_{j}$ satisfies the affineness condition we can prove remarkable results concerning the $\chi\left(\stackrel{\circ}{E}_{I}\right)$, where $E_{I} \subset E_{j}$ and $d \mid N_{i}$ for all $i \in I$.

We first consider the easy case when only one other $E_{i}$ with $d \mid N_{i}$ intersects $E_{j}$ in $C:=E_{i} \cap E_{j}$. If $E_{j}$ satisfies the affineness condition, then $\stackrel{\circ}{E}_{j} \cup \stackrel{\circ}{C}$ is affine and so $H^{k}\left(E_{j},\left(\Psi^{0}\right)^{\varkappa}\right) \cong H_{c}^{k}\left(\stackrel{\circ}{E}_{j} \cup \stackrel{\circ}{C},\left(\Psi^{0}\right)^{\varkappa}\right)=0$ for $k<n$. Then by Poincaré duality and [SGA4 $\frac{1}{2}$, Sommes Trig. 1.19.1] we have that $H^{k}\left(E_{j},\left(\Psi^{0}\right)^{\varkappa}\right)=0$ also for $k>n$.

Now since $\stackrel{\circ}{E}_{j} \cup \stackrel{\circ}{C}$ is affine, its closed subvariety $\stackrel{\circ}{C}$ is also affine and so analogously $H^{k}\left(C,\left(\Psi^{0}\right)^{\varkappa}\right)=H_{c}^{k}\left(\stackrel{\circ}{C},\left(\Psi^{0}\right)^{\varkappa}\right)=0$ for $k \neq n-1$. Using the exact sequence of cohomology with compact support we have the exact sequence

$$
0 \rightarrow H^{n-1}\left(C,\left(\Psi^{0}\right)^{\varkappa}\right) \rightarrow H_{c}^{n}\left(\stackrel{\circ}{E}_{j},\left(\Psi^{0}\right)^{\varkappa}\right) \rightarrow H^{n}\left(E_{j},\left(\Psi^{0}\right)^{\varkappa}\right) \rightarrow 0
$$

and

$$
H_{c}^{k}\left(\stackrel{\circ}{E}_{j},\left(\Psi^{0}\right)^{\varkappa}\right)=0 \text { for } k \neq n .
$$

So there is a remarkable implication

$$
\begin{aligned}
\chi\left(\stackrel{\circ}{E}_{j}\right)=0 & \Rightarrow H_{c}^{n}\left(\stackrel{\circ}{E}_{j},\left(\Psi^{0}\right)^{\varkappa}\right)=0 \Rightarrow H^{n-1}\left(C,\left(\Psi^{0}\right)^{\varkappa}\right)=H_{c}^{n-1}\left(\stackrel{\circ}{C},\left(\Psi^{0}\right)^{\varkappa}\right)=0 \\
& \Rightarrow \chi(\stackrel{\circ}{C})=0 .
\end{aligned}
$$

The following proposition generalizes these ideas.

4.3. Proposition. Fix $E_{j}, j \in S$, with $d \mid N_{j}$ and satisfying the affineness condition (4.1).

(i) For all $I \subset S$ such that $E_{I} \subset E_{j}$ and $d \mid N_{i}$ for all $i \in I$ we have that

$$
\left\{\begin{array}{l}
H^{k}\left(E_{I},\left(\Psi^{0}\right)^{\varkappa}\right)=0 \\
H_{c}^{k}\left(\stackrel{\circ}{E}_{I},\left(\Psi^{0}\right)^{\varkappa}\right)=0
\end{array} \quad \text { for } k \neq n-|I|+1=\operatorname{dim} E_{I} .\right.
$$

In particular $H_{c}^{k}\left(\stackrel{\circ}{E}_{j},\left(\Psi^{0}\right)^{\varkappa}\right)=0$ for $k \neq n$.

(ii) If $\chi\left(\stackrel{\circ}{E}_{j}\right)=0$, then for all $I$ in (i) we have that $\chi\left(\stackrel{\circ}{E}_{I}\right)=0$.

Proof. (i) Denote $B:=\bigcup_{d{ }_{X N}} E_{i}$. The affineness condition implies that all $E_{I} \backslash B$ are affine and hence that

$$
H^{k}\left(E_{I},\left(\Psi^{0}\right)^{\varkappa}\right) \cong H_{c}^{k}\left(E_{I} \backslash B,\left(\Psi^{0}\right)^{\varkappa}\right)=0 \quad \text { for } k \neq \operatorname{dim} E_{I}
$$

by Poincaré duality and [SGA4 $\frac{1}{2}$, Sommes Trig. 1.19.1]. 
Denote $\mathscr{J}:=\left\{I \subset S \mid E_{I} \subset E_{j}\right.$ and $d \mid N_{i}$ for all $\left.i \in I\right\}$ and fix some $I \in \mathscr{J}$. Using repeatedly the Mayer-Vietoris exact sequence or the appropriate spectral sequence we have by (1) that

$$
H^{n-|I|}\left(\bigcup_{\substack{J \supseteq I \\|J|=|I|+1}} E_{J},\left(\Psi^{0}\right)^{\varkappa}\right) \cong \bigoplus_{J \supseteq I} H^{\operatorname{dim} E_{J}}\left(E_{J},\left(\Psi^{0}\right)^{\varkappa}\right)
$$

and

$$
H^{k}\left(\bigcup_{\substack{J \geq I \\|J|=|I|+1}} E_{J},\left(\Psi^{0}\right)^{\varkappa}\right)=0 \quad \text { for } k \neq n-|I| .
$$

Then (1) and (2b) imply the exact sequence

$$
\begin{aligned}
0 & \rightarrow H^{n-|I|}\left(\bigcup_{\substack{J \supseteq I \\
|J|=|I|+1}} E_{J},\left(\Psi^{0}\right)^{\varkappa}\right) \rightarrow H_{c}^{\operatorname{dim} E_{I}}\left(\stackrel{\circ}{E}_{I},\left(\Psi^{0}\right)^{\varkappa}\right) \\
& \rightarrow H^{\operatorname{dim} E_{I}}\left(E_{I},\left(\Psi^{0}\right)^{\varkappa}\right) \rightarrow 0
\end{aligned}
$$

and

$$
H_{c}^{k}\left(\stackrel{\circ}{E}_{I},\left(\Psi^{0}\right)^{\varkappa}\right)=0 \quad \text { for } \quad k \neq \operatorname{dim} E_{I} .
$$

(ii) We have the implications

$$
\begin{aligned}
\chi\left(\stackrel{\circ}{E}_{j}\right)=0 & \Rightarrow H_{c}^{n}\left(\stackrel{\circ}{E}_{j},\left(\Psi^{0}\right)^{\varkappa}\right)=0 \quad \text { by }(3 \mathrm{~b}) \text { with } I=\{j\} \\
& \Rightarrow H^{n-1}\left(\bigcup_{\substack{J \ni j \\
|J|=2}} E_{J},\left(\Psi^{0}\right)^{\varkappa}\right)=0 \quad \text { by }(3 \mathrm{a}) \text { with } I=\{j\} \\
& \Rightarrow \bigoplus_{J \supseteq\{j\}} H^{\operatorname{dim} E_{J}}\left(E_{J},\left(\Psi^{0}\right)^{\varkappa}\right)=0 \quad \text { by }(2 \mathrm{a}) \text { with } I=\{j\} \\
& \Rightarrow H^{n-|I|}\left(\bigcup_{\substack{J \supseteq I \\
|J|=|I|+1}} E_{J},\left(\Psi^{0}\right)^{\varkappa}\right)=0 \quad \text { for any } I \in \mathcal{J} \quad \text { again by }(2 \mathrm{a}) \\
& \Rightarrow H_{c}^{\operatorname{dim} E_{I}}\left(\stackrel{\circ}{E_{I}},\left(\Psi^{0}\right)^{\varkappa}\right)=0 \quad \text { for any } I \in \mathscr{J} \quad \text { by }(3 \mathrm{a}) \\
& \Rightarrow \chi\left(\stackrel{\circ}{E}_{I}\right)=0 \quad \text { for any } I \in \mathscr{J} \quad \text { by }(3 \mathrm{~b}) .
\end{aligned}
$$

4.4. Theorem. Let $E_{i}, i \in T$, be exceptional varieties with $d \mid N_{i}$ for all $i \in T$ and such that $\bigcup_{i \in T} E_{i}$ is connected. If all but eventually one $E_{i}, i \in T$, satisfy the affineness condition (4.1), then we have the implication

$$
\chi\left(\stackrel{\circ}{E}_{i}\right)=0 \quad \text { for all } \quad i \in T \Rightarrow \chi\left(\stackrel{\circ}{E}_{I}\right)=0 \quad \text { for all } \quad \varnothing \neq I \subset T .
$$

Proof. Immediate from Proposition 4.3(ii).

4.5. Conjectural remark. We expect the following to be true if furthermore the resolution $(X, h)$ is required to be some (not yet determined) 'good' resolution, certainly 'without superfluous blowing ups'. Take $d \in \mathbb{N}$ as in (1.5). If 
$\bigcup_{i \in T} E_{i} \subset h^{-1}\{0\}$ is a maximal connected subset of $\bigcup_{i \in S} E_{i}$ with respect to the property that $d \mid N_{i}$ for all $i \in T$, then we have the implication

$$
\chi\left(\stackrel{\circ}{E}_{i}\right)=0 \quad \text { for all } \quad i \in T \quad \Rightarrow \quad \chi\left(\stackrel{\circ}{E}_{I}\right)=0 \quad \text { for all } \quad \varnothing \neq I \subset T .
$$

(4.6) In the next section we will give an application of Theorem 4.4 and Proposition 4.7 below on the topological zeta function and on Igusa's local zeta function. The condition on $d$ in Proposition 4.7 is part of the holomorphy conjecture for those zeta functions, see (5.4) and (5.12).

4.7. Proposition. Suppose that $d$ does not divide the order (as root of unity) of any eigenvalue of monodromy of $f$ at 0. Let $\bigcup_{i \in T} E_{i} \subset h^{-1}\{0\}$ be a maximal connected subset of $\bigcup_{i \in S} E_{i}$ with respect to the property that $d \mid N_{i}$ for all $i \in T$. If all $N_{i}, i \in T$, are mutually different, then $\chi\left(\stackrel{\circ}{E}_{i}\right)=0$ for all $i \in T$.

Proof. Let $F_{0}$ denote the Milnorfiber of $f$ at $0\left(\in \mathbb{A}^{n+1}\right)$. The condition on $d$ implies that for all $k \geqslant 0$ we have

$$
H^{k}\left(F_{0}\right)^{\varkappa}=0
$$

and hence by (1.4) also

$$
\mathbb{H}^{k}\left(h^{-1}\{0\}, R \Psi^{\varkappa}\right)=0 .
$$

Applying the Mayer-Vietoris exact sequence (as in the proof of Lemma 3.2) yields $\mathbb{H}^{k}\left(\bigcup_{i \in T} E_{i}, R \Psi^{\varkappa}\right)=0$ for all $k \geqslant 0$ and so (Proposition 2.2)

$$
\sum_{i \in T} \chi\left(\stackrel{\circ}{E}_{i}\right)=0 .
$$

Consider now for each $r \in \mathbb{N} \backslash\{0\}$ (such that $r d \leqslant \max _{i \in T} N_{i}$ ) its associated character $\varkappa_{r}$ of order $r d$. Again by the condition on $d$ we have for all $k \geqslant 0$ that $H^{k}\left(F_{0}\right)^{\varkappa_{r}}=0$ and we can derive analogously that

$$
\sum_{i \in T_{r}} \chi\left(\stackrel{\circ}{E}_{i}\right)=0,
$$

where $T_{r}$ is any connected component of $\bigcup_{i \in T, r d \mid N_{i}} E_{i}$. Now the equalities (1) to $(r)$ easily imply the stated result, assuming that the $N_{i}, i \in T$, are mutually different.

4.8. Remark. In Proposition 4.7 the condition that all $N_{i}, i \in T$, are different can be weakened to the more general but less elegant condition that for each $r$ and for every connected component $C$ of $\bigcup_{i \in T, r d \mid N_{i}} E_{i}$ we have that $C \backslash\left(\bigcup_{i \in T, r d>N_{i}} E_{i}\right)$ is irreducible.

4.9. Conjectural remark. Again if $(X, h)$ is required to be some 'good' resolution we expect the statement of Proposition 4.7 to be true in general, i.e. without requiring the $N_{i}, i \in T$, to be different.

\section{ApPlicAtion ON ZETA FUnCtions}

(5.1) In the data of (1.1) we denote for any $i \in S$ by $\nu_{i}-1$ the multiplicity of $E_{i}$ in the divisor of $h^{*}\left(d x_{1} \wedge \cdots \wedge d x_{n+1}\right)$; i.e. the canonical divisor on $X$ is $\sum_{i \in S}\left(\nu_{i}-1\right) E_{i}$. 
5.2. Definition [DL, §3]. To $f \in \mathbb{C}\left[x_{1}, \ldots, x_{n+1}\right]$ and $r \in \mathbb{N} \backslash\{0\}$ is associated the topological zeta function

$$
Z_{\text {top }}^{(r)}(s)=\sum_{\substack{I \subset S \\ \forall i \in I: r \mid N_{i}}} \chi\left(\stackrel{\circ}{E}_{I}\right) \prod_{i \in I} \frac{1}{\nu_{i}+s N_{i}}
$$

for $s \in \mathbb{C}$. The remarkable fact that this expression does not depend on the chosen resolution is proved by expressing $Z_{\text {top }}^{(r)}(s)$ as a limit of Igusa's local zeta functions.

(5.3) So when $r$ divides no $N_{i}$ at all then $Z_{\text {top }}^{(r)}(s)$ is holomorphic on $\mathbb{C}$. Now the $N_{i}$ are not intrinsically associated to $f^{-1}\{0\}$; but the order of any eigenvalue of the local monodromy on $f^{-1}\{0\}$ divides some $N_{i}$ (see [A, Theorem 3] and [D4, Lemma 4.6]). The following conjecture of Denef is motivated by this observation.

5.4. Conjecture. If $d \in \mathbb{N} \backslash\{0\}$ does not divide the order (as root of unity) of any eigenvalue of the local monodromy of $f$ at any point of $f^{-1}\{0\}$, then $Z_{\text {top }}^{(r)}(s)$ is holomorphic on $\mathbb{C}$ for all $r$ such that $d \mid r$.

(5.5) For curves $(n=1)$ we actually proved this conjecture (as well as Conjecture 5.12) in [V], using the following crucial result. Suppose that $(X, h)$ is the canonical resolution of $f^{-1}\{0\}$ in $\mathbb{A}^{2}$ and that $d$ satisfies the assumption of Conjecture 5.4. Then a maximal connected subset $\bigcup_{i \in T} E_{i}$ of $\bigcup_{i \in S} E_{i}$ with respect to the property that $d \mid N_{i}$ for all $i \in T$ consists of just one exceptional curve $E_{i_{0}}$, furthermore satisfying $\chi\left({\stackrel{\circ}{E_{i}}}_{i_{0}}\right)=0$. i.e.

Hence for $d \mid r$ such a 'maximal subset' $E_{i_{0}}$ does not contribute to $Z_{\text {top }}^{(r)}(s)$,

$$
\chi\left(\stackrel{\circ}{E}_{i_{0}}\right) \frac{1}{\nu_{i_{0}}+s N_{i_{0}}}=0 \text {. }
$$

Now for $n \geqslant 2$ such a maximal subset $\bigcup_{i \in T} E_{i}$ can be quite arbitrarily complicated and there is certainly no restriction on $|T|$. The following proposition generalizes this noncontribution result to arbitrary dimensions when certain conditions are imposed on the $E_{i}, i \in T$.

5.6. Proposition. Suppose that $d \in \mathbb{N} \backslash\{0\}$ does not divide the order of any eigenvalue of the local monodromy of $f$ at 0 . Let $\bigcup_{i \in T} E_{i} \subset h^{-1}\{0\}$ be a maximal connected subset of $\bigcup_{i \in S} E_{i}$ with respect to the property that $d \mid N_{i}$ for all $i \in T$.

If all $N_{i}, i \in T$, are mutually different and all but eventually one $E_{i}, i \in T$, satisfy the affineness condition (4.1), then $\bigcup_{i \in T} E_{i}$ does not contribute to $Z_{\mathrm{top}}^{(r)}(s)$ for $d \mid r ;$ i.e.

$$
\sum_{\varnothing \neq I \subset T} \chi\left(\stackrel{\circ}{E}_{I}\right) \prod_{i \in I} \frac{1}{\nu_{i}+s N_{i}}=0 .
$$

Proof. Immediate by Proposition 4.7 and Theorem 4.4.

(5.7) We will conclude by the analogue of Conjecture 5.4 and Proposition 5.6 for Igusa's local zeta function. We first introduce this object.

Let $K$ be a finite extension of the field $\mathbb{Q}_{p}$ of $p$-adic numbers, $R$ the valuation ring of $K, P$ the maximal ideal of $R$, and $\bar{K}=R / P$ the residue field 
with cardinality $q$. For $z \in K$ we denote by ord $z \in \mathbb{Z} \cup\{+\infty\}$ its valuation, $|z|=q^{- \text {ord } z}$ its absolute value, and $\operatorname{ac}(z)=z \pi^{- \text {ord } z}$ its angular component, where $\pi$ is a fixed uniformizing parameter for $R$.

Let $g(x) \in K[x], x=\left(x_{1}, \ldots, x_{n+1}\right)$, be a nonconstant polynomial over $K$ and $\kappa: R^{\times} \rightarrow \mathbb{C}^{\times}$a character of $R^{\times}$, the group of units of $R$. (We formally put $\kappa(0)=0$.) To these data one associates Igusa's local zeta function, which is the meromorphic continuation to $\mathbb{C}$ of

$$
Z_{\kappa}(s)=\int_{R^{n+1}} \kappa(\operatorname{ac} g(x))|g(x)|^{s}|d x|
$$

for $\operatorname{Re} s>0$, where $|d x|$ denotes the Haar measure on $K^{n+1}$, normalized such that $R^{n+1}$ has measure 1. Igusa [Ig] showed that it is a rational function of $q^{-s}$.

There is a formula for $Z_{\kappa}(s)$ in terms of an embedded resolution of $g^{-1}\{0\}$ which is similar to the expression of $Z_{\text {top }}^{(r)}(s)$; see Theorem 5.10.

(5.8) From now on we suppose that $f$ is defined over some number field $F$; so we can define Igusa's local zeta function of $f$ with respect to any completion $K$ of $F$. We furthermore suppose that $\kappa$ is induced by a character $\kappa: \bar{K}^{\times}=$ $\mathbb{F}_{q}^{\times} \rightarrow \mathbb{C}^{\times}$of order $d$. (So in particular $d \mid(q-1)$.)

Fixing an embedding of $\mathbb{C}$ in $\mathbb{Q}_{\ell}^{a}$ we can consider $\kappa$ as a character $\kappa: \mathbb{F}_{q}^{\times} \rightarrow$ $\left(\mathbb{Q}_{\ell}^{a}\right)^{\times}$. Since $\mathbb{F}_{q}^{\times}$is a quotient of the arithmetical local monodromy group $G$ of $\mathbb{A}^{1}\left(\mathbb{F}_{q}\right)$ at 0 , we can extend $\kappa$ to $G$ and then consider its restriction to $\mathscr{F}$. So $\kappa$ induces a character $\varkappa: \mathscr{I} \rightarrow\left(\mathbb{Q}_{\ell}^{a}\right)^{\times}$, which we take as the $\varkappa$ of $(1.5)$. See [D4, (2.1)] for more details.

(5.9) We choose the embedded resolution $(X, h)$ of $(1.1)$ such that it is (scheme-theoretically) defined over $K$, and we furthermore suppose that it has tame good reduction mod $P$; see [D1, §2] or [D3, (3.2)]. (If we choose $(X, h)$ to be defined over the number field $F$ itself we have tame good reduction for almost all completions $K$.)

We denote the reduction $\bmod P$ of $f, h, X$ and the $E_{i}, i \in S$, respectively by $\bar{f}, \bar{h}, \bar{X}$ and $\bar{E}_{i}$. Then also the embedded resolution $\left(\bar{X} \otimes \mathbb{F}_{q}^{a}, \bar{h} \otimes \mathbb{F}_{q}^{a}\right)$ of $\bar{f}^{-1}\{0\} \otimes \mathbb{F}_{q}^{a}$ can be considered as a case of the data of (1.1). More precisely the irreducible components of $\bar{h}^{-1}\left(\bar{f}^{-1}\{0\}\right) \otimes \mathbb{F}_{q}^{a}$ are the connected components of the $\bar{E}_{i} \otimes \mathbb{F}_{q}^{a}, i \in S$, and they have multiplicity $N_{i}$ in the divisor of $(\bar{f} \circ \bar{h}) \otimes \mathbb{F}_{q}^{a}$ on $\bar{X}$; see [D1, §§2-3]. A combination of [D2, Theorem 2.2 and Proposition 3.1] and [D4, Lemma 2.3.3] yields the following formula for $Z_{\kappa}(s)$ in terms of these data.

5.10. Theorem. For $f, \kappa$ and $\varkappa$ as in (5.8) we have that

$$
Z_{\kappa}(s)=q^{-(n+1)} \sum_{I \subset S} c_{I} \prod_{i \in I} \frac{q-1}{q^{\nu_{i}+s N_{i}}-1}
$$

with

$$
c_{I}=\sum_{k}(-1)^{k} \operatorname{Tr}\left(\text { Frob }, H_{c}^{k}\left(\stackrel{\circ}{\bar{E}}_{I} \otimes \mathbb{F}_{q}^{a},\left(\Psi^{0}\right)^{\varkappa}\right)\right),
$$

where $\operatorname{Frob} \in \operatorname{Gal}\left(\mathbb{F}_{q}^{a}, \mathbb{F}_{q}\right)$ is the geometric Frobenius.

Remark. We can restrict the summation above to subsets $I$ for which $d \mid N_{i}$ for all $i \in I$, since $\left.\left(\Psi^{0}\right)^{\varkappa}\right|_{E_{l} \otimes \mathbb{F}_{q}^{a}}=0$ for the other $I$ (Lemma 2.1(i)). 
5.11. Theorem. Suppose that $d \in \mathbb{N} \backslash\{0\}$ does not divide the order of any eigenvalue of the local monodromy of $f$ at 0 . Let $\bigcup_{i \in T} E_{i} \subset h^{-1}\{0\}$ be a maximal connected subset of $\bigcup_{i \in S} E_{i}$ with respect to the property that $d \mid N_{i}$ for all $i \in T$.

If all $N_{i}, i \in T$, are mutually different and all but eventually one $\bar{E}_{i} \otimes \mathbb{F}_{q}^{a}, i \in$ $T$, satisfy the affineness condition (4.1), then we have that

$$
\sum_{\varnothing \neq I \subset T} c_{I} \prod_{i \in I} \frac{q-1}{q^{\nu_{i}+s N_{i}}-1}=0 .
$$

Proof. By Proposition 4.7 we have that $\chi\left(\stackrel{\circ}{E}_{i}\right)=0$ for all $i \in T$. Now since each $E_{i}, i \in T$, is proper we have that $\chi\left(\stackrel{\circ}{E}_{i}\right)=\chi\left(\stackrel{\circ}{E}_{I} \otimes \mathbb{F}_{q}^{a}\right)$. Then Proposition 4.3 applied to the $\bar{E}_{i} \otimes \mathbb{F}_{q}^{a}, i \in T$, yields that $\left.H_{c}^{k}\left(\stackrel{\circ}{E}_{I} \otimes \mathbb{F}_{q}^{a},\left(\Psi^{0}\right)^{\varkappa}\right)\right)=0$ for all $\varnothing \neq I \subset T$ and all $k$, so all $c_{I}$ vanish.

(5.11) This result yields some confirmation for the holomorphy conjecture for Igusa's local zeta function.

5.12. Conjecture [D4, (4.3)]. If $f(x)$ is defined over a number field $F \subset \mathbb{C}$, then for almost all completions $K$ of $F$ (i.e. for all except a finite number) we have the following: if the order of $\kappa$ does not divide the order of any eigenvalue of the (complex) local monodromy of $f$ at any complex point of $f^{-1}\{0\}$, then $Z_{\kappa}(s)$ is holomorphic on $\mathbb{C}$.

\section{REFERENCES}

[A] N. A'Campo, La fonction zeta d'une monodromie, Comment. Math. Helv. 50 (1975), 233-248.

[B] J.-L. Brylinski, Transformations canoniques, dualité projective, théorie de Lefschetz, transformations de Fourier et sommes trigonométriques, Astérisque, vol. 140-141, Soc. Math. France, Paris, 1986.

[D1] J. Denef, On the degree of Igusa's local zeta function, Amer. J. Math. 109 (1987), 991-1008.

[D2] Local zeta functions and Euler characteristics, Duke Math. J. 63 (1991), 713-721.

[D3] _ Report on Igusa's local zeta function, Sém. Bourbaki 741, Astérisque, vol. 201203, Soc. Math. France, Paris, 1991, pp. 359-386.

[D4] - Degree of local zeta functions and monodromy, Compositio Math. 89 (1994), 207-216.

[DL] J. Denef and F. Loeser, Caractéristiques d'Euler-Poincaré, fonctions zeta locales, et modifications analytiques, J. Amer. Math. Soc. 5 (1992), 705-720.

[H] H. Hironaka, Resolution of singularities of an algebraic variety over a field of characteristic zero, Ann. of Math. (2) 79 (1964), 109-326.

[Ig] J. Igusa, Complex powers and asymptotic expansions I, J. Reine Angew. Math. 268/269 (1974), 110-130; II, 278/279 (1975), 307-321.

[II] L. Illusie, Autour du théorème de monodromie locale, prépublication 92-65 de l'Université de Paris-Sud, 1992.

[M] J. Milnor, Singular points of complex hypersurfaces, Princeton Univ. Press, 1968.

[S] T. Saito, $\epsilon$-factor of a tamely ramified sheaf on a variety, Invent. Math. 113 (1993), 389-417.

[SGA4 $\frac{1}{2}$ ] P. Deligne, Cohomologie étale, Lecture Notes in Math., vol. 569, Springer, 1977. 
[SGA7] A. Grothendieck, P. Deligne and N. Katz, Groupes de monodromie en géométrie algébrique I, Lecture Notes in Math., vol. 288, Springer, 1972; II, vol. 340, 1973.

[V] W. Veys, Holomorphy of local zeta functions for curves, Math. Ann. 295 (1993), 635-641.

K. U. Leuven, Departement Wiskunde, CelestijnenlaAn 200B, B-3001 Leuven, Belgium E-mail address: willem=veys\%alg\%wis@cc3.kuleuven. ac.be 